\title{
Satellite image fusion using fuzzy logic
}

\author{
Suda KUMARASWAMY \\ Department of IT, VNRVJIET, Hyderabad, India \\ email: kumaraswamy_it@vnrvjiet.ac.in \\ Dammavalam SRINIVASA \\ $\mathrm{RAO}$ \\ Department of IT, VNRVJIET, \\ Hyderabad, India \\ email: \\ srinivasarao_d@vnrvjiet.ac.in \\ Nuthanapati NAVEEN \\ KUMAR \\ CSE department, SIT,JNTU \\ Hyderabad, India \\ email: naveen.cse.mtech@jntuh.ac.in
}

\begin{abstract}
Image fusion is a method of combining the Multispectral (MS) and Panchromatic (PAN) images into one image contains more information than any of the input. Image fusion aim is to decrease unknown and weaken common data in the fused output image at the same time improving necessary information. Fused images are helpful in various applications like, remote sensing, computer vision, biometrics, change detection, image analysis and image classification. Conventional fusion methods are having some side effects like assertive spatial information and uncertain color information is an usually the problem in PCA and wavelet transform based fusion is a computationally in depth process. In order to overcome these side effects and to propose alternative soft computing fusion approach for conventional fusion methods we exploit image fusion using fuzzy logic technique to fuse two source images obtained from different sensors to enhance both spectral and spatial information. The proposed work here further compared with two common fusion methods like, principal component analysis (PCA) and wavelet transform along with quality assessment metrics. Exploratory outputs demonstrated in
\end{abstract}

Computing Classification System 1998: I.4.3, I.4.9

Mathematics Subject Classification 2010: 68U10

Key words and phrases: Fusion, MS, PAN, Fuzzy Logic, PCA, Wavelet Transform 
order that fuzzy based image fusion technique can actively retains more information compared to PCA and wavelet transform approaches while enhancing the spatial and spectral resolution of the satellite images.

\section{State-of-the art}

The coordinates in the small frequency region with high incisiveness core dimensions are chosen as coefficients of the output image, and a most adjacent intensity stationed fusion method is presented to choose giant frequency coordinates [3]. In [15], novel image fusion approach for confining image sensor network is presented where the sharpen density of the fresh compressive value are not acquired from the arbitrary specimen data still in distinction to the chosen Hadamard conjoined with whatever can additionally be generated against constrict imaging process adequately. Fuzzy based fusion approach compared with discrete wavelet transform (DWT) and weighted average DWT using Genetic algorithm (GA) approaches and shown that fuzzy based image fusion technique out performs DWT and DWT using GA approaches. Image fusion using Pulse-Coupled Neural Network (PCNN) is proposed where input images are flattened through scrambled block Hadamard ensemble (SBHE) in compressed domain and local standard variance is input to drive PCNN and coefficients with huge ignited times are chosen as the fusion coefficients. Later fusion coefficients are whipped by sliding window in order to avoid blocking effect [16]. In [10], image fusion using fuzzy logic and neuro fuzzy logic approaches are compared and concluded that in some cases fuzzy based fusion results gave better results in some other cases neuro fuzzy based fusion generated better results. . A new method of satellite image fusion have been build on Otsu's Multi-thresholding approach in two stages, i) shearlet transform is used Panchromatic and multi-spectral image distinctly, ii) the revised low frequency sub-band shearlet coefficients obtained from shearlet transform are composed by the Otsu's Multi-thresholding approach and choose most lowpass band naturally [2]. A innovative multifocus image fusion approach [17] built on human visual system and neural network back propagation given with three facets which echo brightness of a pixel are extracted first and used to train a BP neural network to decide the clarity pixel. Those pixels are then used to build the initial fused image. Later the focused regions are identified by calculating the coincidence in mid of satellite images and the first time fused image proceed by morphological operations and the final fused image is attained by applying a fusion rule for those concentrated regions. In [8], novel 
fusion method is introduced to invent full utilization of structural compactness for fusion of the common and structured layers. In [4], authors demonstrated a new fusion technique where it separates the input image decomposition technique into two consecutive filtrated activities by applying spectral factorization filter. The concrete image fusion attain after involution along with the early filter couple. Its important lower guide volume directed to the miniaturize of the undesirable expansion of conjoined values about overlaying image peculiarities. In [6], a technique proposed for straight virtue evaluation of fusion process placed on the assessment of triple major elements of output image quality like diversity storage, incisiveness and anatomy preservation. Intuitive analysis is postured to construct a database with fusion to evaluate the achievement of the fusion process.

\section{Wavelet transform based image fusion}

In [7], wavelet transform applied a structure in which a input image is decomposed, where individual plain correlating to a mean decision, or reduced periodicity strip. Fusion using this methodology is a category of input model that can allow the density contended about the input at appropriate moment. The framework for wavelt based fusion illustrated in Fig. 1.

Algorithm for wavelet based image fusion [5]

1. Take two input images, K1 and K2 to be fused.

2. Apply the wavelet decomposition process on the two input images.

3. Employ the pixel based approach for similarity whatever contain fusion situated on considering the higher valued image pixels from likeness of source images K1and K2.

4. Placed on higher valued image pixels ,a binate determination map is produced and it gives the decision rule for the conduction fusion of two input images K1and K2.

5 . The output fused transform interrelated to similarity over higher selection pixel rule is generated.

6. Connecting of fused resemblance and particulars produces the new coefficient matrix.

7. Execute the inverse wavelet transform process to build the output fused image. 


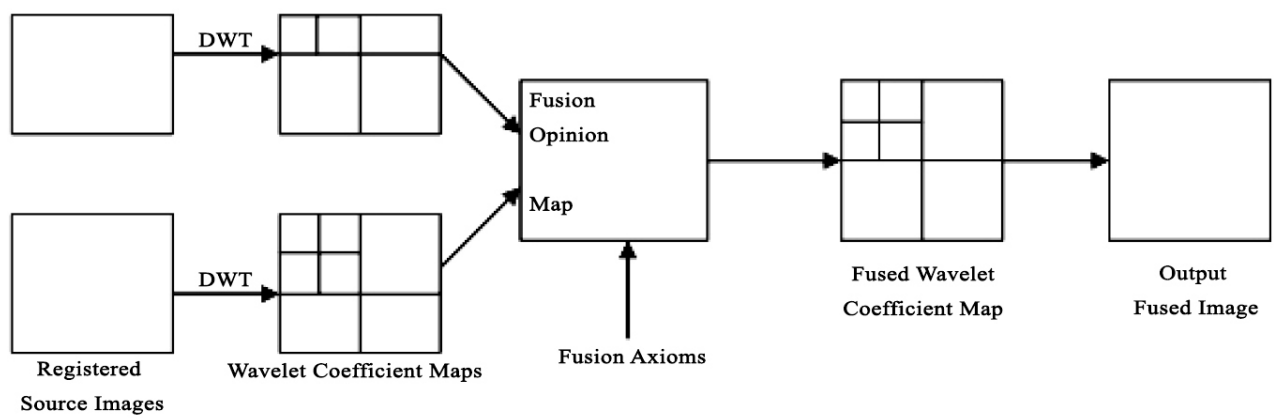

Figure 1: The generic structure for wavelet transform based image fusion

\section{Principal component analysis based image fusion}

A numerical concept that transforms a number of correlated input variables into a numeral unassociated variables through the Principal Component Analysis (PCA). Algorithm steps in PCA concept is as follows

Algorithm steps:

1. Input image are transformed in to column vectors initially.

2. Covariance matrix is calculated from two column vectors.

3. Compute eigenvalues and the corresponding eigenvectors.

4. Normalize both the characteristic values and characteristic vectors.

5. Through fusion process on two scaled matrices, final fused image matrix is generated.

We consider the input images denoted by $A(i, j)$ and $B(i, j)$ and convert these images in to equivalent dual column vectors and means are subtracted. The dimension of the output is $\mathrm{m} \times 2$, here $\mathrm{m}$ is the magnitude of the image. The eigenvalues and conform eigenvectors considering output is calculated also compute the eigenvectors correlated to the greater eigenvalues. P1 and P2 are normalized components computed from covariance matrix to obtain eigenvector and the fused image is obtained from it [12].

\section{Fuzzy logic based image fusion}

Two registered input images are used in the fusion process. Fuzzy logic properties are utilized to perform fusion. An innovative image fusion for in multi-view over the-wall radar imaging system to compute the variation among pixels us- 
ing a local operator and concluded that method performs well compared to conventional fusion approaches [13]. A different method is proposed to fuse images by utilizing maximum, minimum operations in intuitionist fuzzy sets (IFSs). Entropy metric is used to generate the most favorable value of the parameter in membership functions. Later resulting IFIs are decomposed into image sections and the correlated sections of the images are combined by computing blackness and whiteness of the blocks [1]. An algorithm for image fusion is conferred stands on fuzzy logic and wavelet transform and evaluate the pixel-level image fusion approaches, and focus on a technique based on the discrete wavelet transform and fuzzy logic approaches. As part of the fusion process two fuzzy relations are determined and predicted the essence of each one wavelet coefficient with fuzzy hypothesis. Based on the priority of coefficients, the weighting average coefficients were computed. Finally the fused image is obtained through inverse wavelet transform operation [18]. A new image fusion technique based on fuzzy logic and Discrete Wavelet Transform (DWT). The fuzzy membership functions and fuzzy rules are composed properly to perfect adaption for the fusion of multifocus images. DWT has been utilized to enhance the attainment as fuzzy logic is practiced at every stage of DWT to perform fusion on similar coefficients [9].

\subsection{Image processing with fuzzy logic}

Image processing with fuzzy approach has triple essential steps. The encoding of input image and decoding the fused image are important stages that are get ready to operate the original image using fuzzy techniques. After the inputs are converted to the associates uniform adapted procedures update the fellows weights [11].

\subsection{Procedure for fuzzy based image fusion}

Fuzzy rules, Membership Functions (MSF) are utilized in the fusion process [12]

Axiom-1: $\quad\left[I / P_{1} i s M S F_{3}\right]$ or $\left[I / P_{2} i s M S F_{3}\right] \rightarrow\left[O / P_{1} i s M S F_{2}\right]$

Axiom-2: $\quad\left[I / P_{1} M S F_{1}\right]$ or $\left[I / P_{2} i s M S F_{3}\right] \rightarrow\left[O / P_{1} i s M S F_{1}\right]$

Axiom-3: $\quad\left[I / P_{1} M S F_{3}\right]$ or $\left[I / P_{2} i s M S F_{2}\right] \rightarrow\left[O / P_{1} i s M S F_{3}\right]$ 


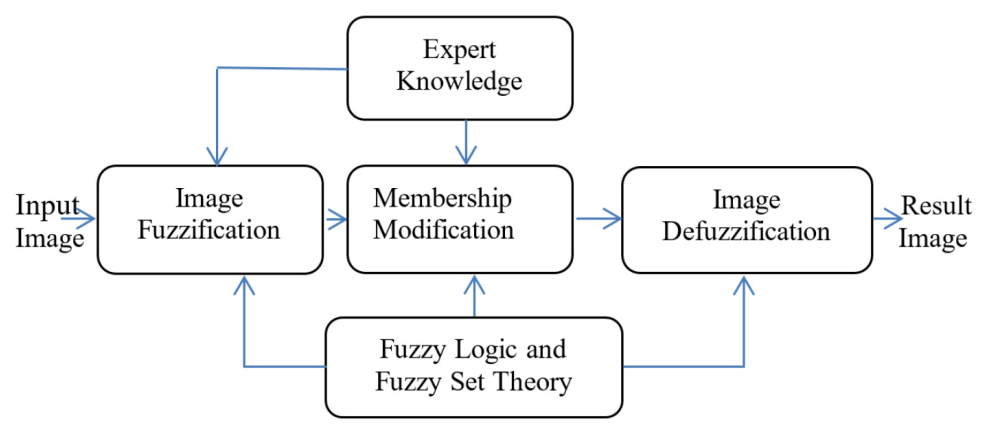

Figure 2: Block diagram for fuzzy image processing

Axiom-4: $\quad\left[I / P_{1} M S F_{2}\right]$ or $\left[I / P_{2} i s M S F_{2}\right] \rightarrow\left[O / P_{1} i s M S F_{2}\right]$

Axiom-5: $\quad\left[I / P_{1} M S F_{2}\right]$ or $\left[I / P_{2} i s M S F_{2}\right] \rightarrow\left[O / P_{1} i s M S F_{2}\right]$

Axiom-6: $\quad\left[I / P_{1} M S F_{1}\right] \operatorname{or}\left[I / P_{2} i s M S F 2\right] \rightarrow\left[O / P_{1} i s M S F_{1}\right]$

Sequence of steps in fuzzy logic based image fusion as follows [15].

1. Get the first image in K1 and its size (rows: row1, columns: col1).

2. Get the second image in K2 and its size (rows:row2, columns: col2).

3. Images $\mathrm{K} 1$ and $\mathrm{K} 2$ are in matrix form and each pixel value is in between 0-255. Apply Grey Colormap.

4. Select two input images which are in same size.

5. Transform two input images in column matrix which has $\mathrm{S}=$ rowl*coll entries.

6. Prepare a fis (Fuzzy) file, which has two input images.

7. Determine fuzzy membership functions for input images to be fused by adapting the membership functions

8. Prepare fuzzy rules for the fusion process

9. For num=l to $\mathrm{S}$ in steps of one, utilize fuzzification step by employing the fuzzy rules on input images 
10. Transform the column form to matrix form and display the output fused image.

\section{$5 \quad$ Quality metrics}

Quality assessment parameters are applied to assess the fused image obtained from the fusion operation.

\subsection{Quality index (QI)}

QI calculates the affinity between two images (A \& B) and QI is equivalent to 1 if both the images are exact [11]

$$
Q I=\frac{m_{a b} 2 x y 2 m_{a} 2 m_{b}}{m_{a} m_{b} x^{2}+y^{2} m_{a}^{2}+m_{b}^{2}}
$$

where input images (A \& B) mean values are denoted by $\mathrm{x}, \mathrm{y}$ and variance, covariance of images are denoted by $, M_{a}^{2}, M_{b}^{2}$, and $M_{a b}$, QI indicates the amount of the information presented in reference image has been converted into the output fused image. The ideal value 1 indicates fused image and reference images are similar.

\subsection{Mutual information measure (MIM)}

MIM contains the mutual information between $A(i, j)$ and $B(i, j)$ input images,

$$
I_{A B}=\sum_{x, y} P_{A B}(x, y) \log \frac{P_{A B}(x, y)}{P_{A}(x), P_{B}(y)}
$$

where, $P_{A}(\mathrm{x})$ and $P_{B}(\mathrm{y})$ are the probability in the individual images, and $\left.P_{A B}(\mathrm{x}, \mathrm{y})\right)$ is joint probability, higher value indicates better fused image quality.

\subsection{Fusion factor (FF)}

Two input images are A,B and $\mathrm{F}$ is their fused image [14], then

$$
F F=I_{A F}+I_{B F}
$$

where MIM values between input images and used image are denoted by $I_{A F}$ and $I_{B F}$ respectively. Maximum value of $\mathrm{FF}$ denotes that output fused image 
consists of reasonably superior amount of information existent in both the images.

\subsection{Fusion symmetry (FS)}

FS is a notion of the intensity of equivalence in the image content of two images.

$$
I_{A B}=a b s\left(\frac{I_{A F}}{I_{A F}+I_{B F}}-0.5\right)
$$

Lower FS value indicates that the fused image obtains features from both source images.

\subsection{Fusion index (FI)}

Based on two fusion metrics, fusion symmetry and fusion factor the fusion index, FI is calculated as

$$
F I=\frac{I_{A F}}{I_{B F}}
$$

where IAF denotes mutual information between MS image and fused image and IBF is the mutual information between PAN image and fused image. The quality of fusion approach indicated by the degree of fusion indEx.

\subsection{Root mean square error (RMSE)}

The RMSE calculates the intensity of the pixel difference obtained from the fusion process.

$$
R M S E=\sqrt{\frac{1}{M N} \sum_{i=1}^{M} \sum_{j=1}^{N}(R(i, j)-F(i, j))}
$$

lower RMSE value indicates better fusion approach.

\subsection{Peak signal to noise ratio (PSNR)}

PSNR can be determined by

$$
P S N R=20 \log _{10}\left(\frac{G^{2}}{M S E}\right)
$$

where $G$ is the intensity of gray in the fused image, maximum PSNR value denotes better fused image quality. 
Table 1: Quality metrics for outputs obtained from conventional and proposed approaches

\begin{tabular}{lllllllll}
\hline Approach & QI & FF & FS & FI & MIM & RMSE & PSNR & E \\
\hline Wavelet & & & & & & & & \\
(Ex. 1) & 0.9463 & 3.7629 & 0.0529 & 1.0779 & 1.6554 & 62.5529 & 11.2425 & 7.3415 \\
(Ex. 2) & 0.8550 & 3.7832 & 0.0218 & 0.8938 & 1.9775 & 18.8999 & 22.3648 & 7.1134 \\
(Ex. 3) & 0.9358 & 0.9278 & 0.0128 & 1.0527 & 0.4520 & 20.7690 & 21.7825 & 7.1454 \\
(Ex. 4) & 0.9425 & 1.2222 & 0.0391 & 1.0882 & 0.5848 & 13.8566 & 25.2977 & 7.2511 \\
& & & & & & & & \\
\hline & & & & & & & & \\
PCA & & & & & & & & \\
(Ex. 1) & 0.9450 & 1.5650 & 2.7964 & 0.0765 & 1.2913 & 9.4765 & 27.9806 & 7.2721 \\
(Ex. 2) & 0.9876 & 1.5890 & 3.2885 & 0.0110 & 0.7932 & 17.440 & 19.9290 & 7.3228 \\
(Ex. 3) & 0.9353 & 1.2194 & 0.0149 & 0.9422 & 0.8341 & 25.0039 & 20.2047 & 7.4532 \\
(Ex. 4) & 0.9397 & 1.5546 & 0.0402 & 1.1749 & 1.3800 & 25.6658 & 13.3336 & 7.4213 \\
& & & & & & & & \\
\hline & & & & & & & & \\
Fuzzy & & & & & & & & \\
(Ex. 1) & 0.9689 & 5.5324 & 0.2552 & 3.2875 & 4.3345 & 12.2001 & 23.8336 & 7.3865 \\
(Ex. 2) & 0.9955 & 8.6207 & 0.0498 & 1.2826 & 3.8823 & 16.8785 & 23.2336 & 7.3577 \\
(Ex. 3) & 0.9470 & 1.2919 & 0.0092 & 1.3133 & 0.9870 & 20.3423 & 21.8432 & 7.4322 \\
(Ex. 4) & 0.9431 & 1.5948 & 0.0384 & 1.2404 & 1.4086 & 21.7479 & 20.0220 & 7.4391 \\
& & & & & & & & \\
\hline
\end{tabular}

\subsection{Entropy (E)}

Entropy represents the quality of the source image. Entropy is a amount of volatility that can be used to discrminate the texture of the input image

$$
E=-\sum p * \log _{2}(p)
$$

where $\mathrm{p}$ represents the scatter diagram count.

\section{Results analysis}

In this paper, we fused a MS and PAN images using our fuzzy based fusion algorithm. Ex. 1, Ex. 2 MS and PAN images, Hyderabad captured from LISS III. Ex. 2 images are collected from http://www.metapix.de/examples_r.htm [8], Ex. 3 images are taken from the NRSC test samples.

The fuzzy logic based image fusion process has been carried out using Matlab 10.0. In order to implement fuzzy based image fusion required fuzzy membership functions and fuzzy rules are tuned and determined precisely. Because of the potentiality of the fuzzy logic, similarity between fused image and reference image denoted by IQI value $(0.9689,0.9955,0.9470$ and 0.9431$)$ obtained 


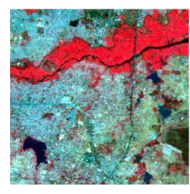

Ex. 1: (a)

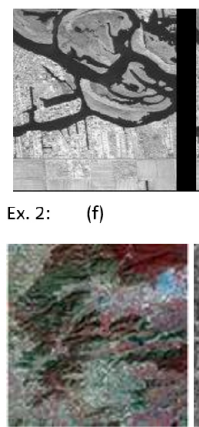

Ex. 3: (k)

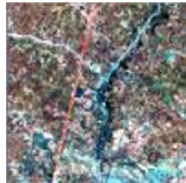

Ex. 4: (p)

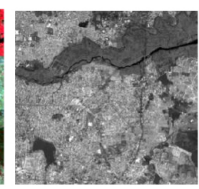

(b)

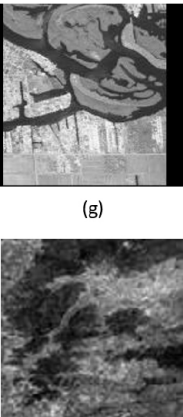

(I)

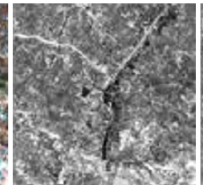

(q)

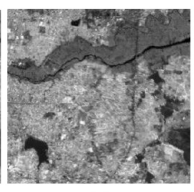

(c)

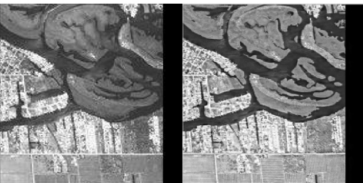

(h)

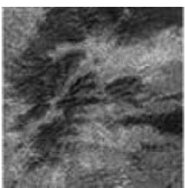

(m)

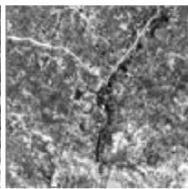

(r)

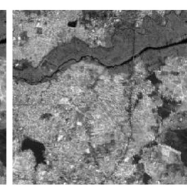

(d)

(i)

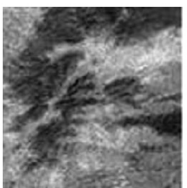

(n)

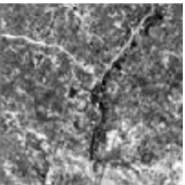

(s)

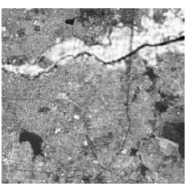

(e)

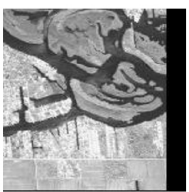

(j)

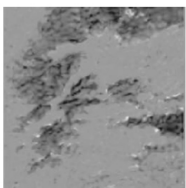

(0)

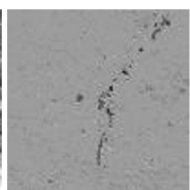

(t)

Figure 3: Case study: (a), (b), (f), (g),(k),(l),(p),(q): input images; (c), $(\mathrm{h}),(\mathrm{m}),(\mathrm{r})$ are output images from wavelet transform and (d), (i),(n),(s) are fused from PCA and (e), (j), (o) and (t) fused images obtained from proposed fuzzy approach. 
from fuzzy based fusion having better values compared to IQI values $(0.9463$, $0.8850,0.9358$ and 0.9425$)$ from wavelet based fusion and IQI values $(0.9450$, $0.9876,0.9353$ and 0.9357 ) obtained from PCA based fusion approaches respectively. Typical assessment parameters like fusion factor values (5.5324, $8.6207,1.2919$ and 1.5948 ) and fusion index values $(3.2875,1.2826,1.3133$ and 1.2404) are also having better values indicates that proposed fuzzy based image fusion approach enhanced the fusion quality compared to traditional fusion methods. Higher values for FF obtained from proposed method indicates that information contained in the fuzzy based fused image is more possessing extremely good quality results compared with the wavelet transform and PCA based fusion approaches. Higher values for FI metric generated from the fuzzy based fusion approach indicates that fusion degree is higher for proposed method compared to other methods mentioned. Higher value for PSNR and Entropy obtained from proposed fuzzy based fusion method indicates that amount of information in the fused image is high compared to wavelet and PCA based fusion approaches respectively. Table 1 demonstrated that proposed fuzzy based fusion approach has exhibited conditionally more effective in QI, MIM and Entropy values while improving spectral and spatial information as well. Substantial variances are generated through fuzzy based fusion with lower values for RMSE, FS and having greater values for FF, FI and PSNR assessment metrics. Hence it is concluded from experimentation outputs that image fusion using fuzzy logic scheme out performs conventional wavelet transform and PCA based fusion approaches.

\section{Conclusion and future work}

In this paper, fuzzy logic based image fusion for satellite images obsolete conferred. The result analysis certainly proves that the proposed fuzzy logic based fusion provides a huge progress on the attainment of the process. The proposed approach can be applied iteratively and also applied to all categories of images and to integrate conclusive assessment parameter of different image fusion approaches. Classification of fused images may also improve accuracy in remote sensing objectives. So it has been examined from experimental outcomes that proposed fuzzy based image fusion algorithm conserve superior spatial and spectral information and also improved visual essence compared to conventional fusion methods, wavelet trans from and PCA methods. 


\section{References}

[1] P. Balasubramaniam, V. P. Ananthi: Image fusion using intuitionistic fuzzy sets, Elsever Journal of Information Fusion 20 (2014) 21-30. $\Rightarrow 245$

[2] B. Biswas, K. N. Dey, A. Chakrabarti, Remote sensing image fusion using multithreshold Otsu method in Shearlet domain, Procedia Computer Science 57, (2015) 554-562. $\Rightarrow 242$

[3] Y. Chen, Z. Qin, PCNN-based image fusion in compressed domain, Mathematical Problems in Engineering, Vol. 2015. $\Rightarrow 242$

[4] A. Ellmauthaler, C. L. Pagliari, E. A. B. da Silva, Multiscale image fusion using the undecimated wavelet transform with spectral factorization and nonorthogonal filter banks, IEEE Transactions on Image Processing, 22, 3 (2013) 10051017. $\Rightarrow 243$

[5] D. L. A. Godse, D. S. Bormane, Wavelet based image fusion using pixel based maximum selection rule, International Journal of Engineering Science and Technology, 3, 7, (2011) 5572-5557. $\Rightarrow 243$

[6] R. Hassen, Z. Wang, M. M. A. Salama, Objective quality assessment for multiexposure multifocus image fusion, IEEE Transactions on Image Processing $\mathbf{2 4}$, 9 (2015) 2712-2724. $\Rightarrow 243$

[7] K. Kannan, S. A. Perumal, K. Arulmozhi, Performance comparision of various levels of fusion of multi-focused images using wavelet transform, I. J. Computer Applications, 1, 6 (2010) 71-78. $\Rightarrow 243$

[8] S. Li, X. Kang, J. Hu, Image fusion with guided filtering, IEEE Transactions on Image Processing, 22, 7 (2013) 2864-2875. $\Rightarrow 242,249$

[9] A. N. Myna, J. Prakash, A novel hybrid approach for multi-focus image fusion using fuzzy logic and wavelets, International Journal of Emerging Trends and Technology in Computer Science, (IJETTCS), 3, (2014) 131-138. $\Rightarrow 245$

[10] D. S. Rao, M. Seetha, M. H. M. Krishna Prasad, Comparison of fuzzy and neuro fuzzy image fusion techniques and its applications, International Journal of Computer Applications, 43, 20 (2012) 31-37. $\Rightarrow 242$

[11] D. S. Rao, M. Seetha, M. H. M. Krishna Prasad, Quality assessment of pixellevel image fusion using fuzzy logic, International Journal on Soft Computing, 3, 1, (2012) 13-25. $\Rightarrow 245,247$

[12] D. S. Rao, M. Seetha, M. H. M. Krishna Prasad, Novel approach for iterative image fusion using fuzzy and neuro fuzzy logic, International Journal of Geoinformatics 11, 2 (2015) 29-39. $\Rightarrow 244,245$

[13] C. H. Seng, A. Bouzerdoum, F. H. C. Tivive, M. G. Amin, Fuzzy logic-based image fusion for multi-view through-the-wall radar, Int. Conf. Digital Image Computing: Techniques and Applications (DICTA), 2010, pp. 423-428. $\Rightarrow 245$

[14] M. Seetha, I. V. Murali Krishna, B. L. Deekshatulu, Data fusion performance analysis based on conventional and wavelet transform techniques, IEEE Proceedings on Geoscience and Remote Sensing Symposium 4 (2005) 2842-2845. $\Rightarrow 247$ 
[15] C. Yang, B. Yang, Efficient compressive multi-focus image fusion, Journal of Computer and Communications, 2 (2014) 78-86. $\Rightarrow 242,246$

[16] Y. Yang, S. Huang, J. Gao, Z. Qian: Multi-focus image fusion using an effective discrete wavelet transform based algorithm, Measurement Science Review, 14, 2 (2014) 102-108. $\Rightarrow 242$

[17] Y. Yang, W. Zheng, S. Huang, Effective multifocus image fusion based on HVS and BP neural network, The Scientific World Journal, 2014, Article ID 281073, 10 pages. $\Rightarrow 242$

[18] M. Zhu, Y. Yang, A new image fusion algorithm based on fuzzy logic, International Conference on Intelligent Computation Technology and Automation, (ICICTA) 2008, pp. 83-86. $\Rightarrow 245$

Received: July 8, 2016 • Revised: November 27, 2016 